\title{
Short communication: Assessment of disbudding pain in dairy calves using nonlinear measures of heart rate variability
}

\author{
C. J. Byrd, ${ }^{1 *}+$ B. A. Craig, ${ }^{2}$ S. D. Eicher, ${ }^{3}$ J. S. Radcliffe, ${ }^{1}$ and D. C. Lay Jr. ${ }^{3}$ \\ ${ }^{1}$ Department of Animal Sciences, Purdue University, West Lafayette, IN 47907 \\ ${ }^{2}$ Department of Statistics, Purdue University, West Lafayette, IN 47907 \\ ${ }^{3}$ USDA-Agricultural Research Service Livestock Behavior Research Unit, West Lafayette, IN 47907
}

\section{ABSTRACT}

The purpose of this study was to evaluate whether pain-related stress caused by disbudding could be detected using nonlinear measures of heart rate variability (HRV). Twenty-five female Holstein calves (4-7 wk of age) were randomly assigned to 1 of 3 treatments: (1) sham disbud (SHAM; $\mathrm{n}=9$ ), (2) disbud with lidocaine-meloxicam pain mitigation (MED; $\mathrm{n}=8$ ), or (3) disbud without pain mitigation (NoMED; $\mathrm{n}=8$ ). Heart rate variability (sample entropy, percentage determinism, percentage recurrence, or mean length of diagonal lines in a recurrence plot) was recorded on $\mathrm{d}-1,0,1,3$, and 5 relative to the experimental procedure, with disbudding taking place on $\mathrm{d} 0$. The short-term detrended fluctuation analysis scaling exponent was greater in MED calves than in SHAM calves, indicating a greater stress response to the disbudding procedure regardless of pain mitigation. These results indicate that calves in the MED group may have experienced pain-related stress as a result of the disbudding procedure. The remaining nonlinear HRV measures did not differ by treatment. Future research on this topic should address additional potential confounding factors, such as the effect of pain-mitigating drugs on autonomic function or the influence of the autonomic nervous system on wound healing, that may prohibit HRV measurement as an indicator of disbudding pain severity.

Key words: dairy calf, disbudding, heart rate variability, pain, stress

\section{Short Communication}

Disbudding, or the removal of the horn buds, is a common procedure performed on dairy calves to in-

Received November 5, 2018.

Accepted May 13, 2019.

*Corresponding author: christopher.byrd@ndsu.edu

$\dagger$ Current address: Department of Animal Sciences, North Dakota State University, Fargo 58108. hibit horn growth. In the United States, $94 \%$ of dairies perform some type of disbudding or dehorning procedure (USDA, 2018). Approximately $50 \%$ of calves born annually are disbudded with a hot-iron dehorner, and of those, approximately $70 \%$ go without analgesic or anesthetic treatment for management of pain (USDA, 2018). It is well established that disbudding or dehorning without pain management increases plasma cortisol concentrations (Petrie et al., 1996; Heinrich et al., 2009), increases sensitivity to pressure applied to the horn bud area (Heinrich et al., 2010), increases heart and respiration rates (Stewart et al., 2008; Heinrich et al., 2009), and alters behavior for 12 to $44 \mathrm{~h}$ (Faulkner and Weary, 2000; Heinrich et al., 2010); however, the heart rate variability (HRV) response to disbudding is less clear.

Heart rate variability, or the change in time interval between adjacent heartbeats, is a commonly used proxy measure of the autonomic stress response in dairy cattle. The sympathetic and parasympathetic branches of the autonomic nervous system innervate the heart and, in general, act in an antagonistic manner in response to a stressor (generally, the sympathetic branch increases heart rate and the parasympathetic branch decreases heart rate; Rea, 2016). As a result, changes to the interval between adjacent heartbeats over a period of time have been used in a variety of ways to elucidate information regarding the state of the autonomic nervous system in response to different on-farm stressors, including painful procedures such as disbudding (Stewart et al., 2008, 2009).

Some evidence has shown that the low frequency to high frequency ratio (LF/HF; an HRV measure commonly used to evaluate sympatho-vagal balance) is increased immediately following disbudding in calves disbudded without pain mitigation (Stewart et al., 2008 ) and 2 to $3 \mathrm{~h}$ later in calves given a lidocaine cornual nerve block before the procedure (Stewart et al., 2009). This indicates that sympathetic activity increases in response to immediate disbudding pain and after pain mitigation has diminished. Additionally, the 
Table 1. Definitions of heart rate (HR) variability (HRV) parameters

\begin{tabular}{|c|c|}
\hline Parameter & Practical definition \\
\hline & $\alpha_{1}>0.5:$ data are negatively correlated \\
\hline & $\alpha_{1}=0.5:$ data are random and do not have long-range correlations \\
\hline & Long-range correlations indicate increased self-similarity of the HRV data at different time lengths. \\
\hline & $\begin{array}{l}\text { Values close to zero are believed to indicate healthy physiological complexity and low stress, whereas } \\
\text { values around } 1.5 \text { and } 0.5 \text { are indicative of reduced physiological complexity and greater stress } \\
\text { (Goldberger et al.. 2002a). }\end{array}$ \\
\hline Recurrence (\%REC), \% & $\begin{array}{l}\text { Determined using recurrence quantification analysis, the percentage of recurrent points (within some } \\
\text { radius, r) in the recurrence plot. Greater values indicate increased HR regularity and greater stress } \\
\text { (Mohr et al., 2002; Hagen et al., 2005). }\end{array}$ \\
\hline
\end{tabular}

root mean square of successive differences (a measure of parasympathetic function) is lowered immediately following disbudding in calves not given pain mitigation, indicating decreased parasympathetic activity (Stewart et al., 2009). Taken together, these results suggest that calves experience pain from disbudding. However, very few additional changes to HRV have been demonstrated as a result of disbudding.

One potential reason for this may be that studies focused on HRV following disbudding have only investigated traditional linear HRV measures that evaluate changes in mean, variance, and the frequency spectra of the HRV data. However, changes in HRV over time often exhibit nonlinear properties that may be better measured using nonlinear analyses capable of evaluating correlational, structural, and organizational aspects of the data (Goldberger et al., 2002b; Billman, 2013). Published data on nonlinear HRV in dairy cattle are limited (see Mohr et al., 2002; Hagen et al., 2005; Erdmann et al., 2018), and, to the authors' knowledge, only 1 study has focused on the nonlinear HRV response to painful procedures or states (Kovács et al., 2015). Therefore, the objective of this study was to evaluate nonlinear measures of HRV following disbudding in Holstein heifer dairy calves. Additionally, because disbudding has been shown to alter calf behavior for at least $44 \mathrm{~h}$ postdisbudding (Heinrich et al., 2010), nonlinear HRV was evaluated at 3 additional time points over a 5 -d postdisbudding period.

We hypothesized that calves disbudded without pain mitigation would exhibit altered nonlinear HRV properties indicative of greater stress compared with calves that were disbudded with pain mitigation or sham disbudded. Specifically, we predicted that calves disbudded without pain mitigation would have lower sample entropy (SampEn; Table 1), greater percentage determinism (\%DET; Table 1), percentage recurrence (\%REC; Table 1), and mean length of diagonal lines in a recurrence plot (Lmean; Table 1), and lower signal complexity (as measured by short-term detrended fluctuation analysis; $\mathbf{D F A} \boldsymbol{\alpha}_{1}$; Table 1) compared with calves that received pain mitigation before disbudding or were sham disbudded.

All experimental procedures were approved by the Purdue University Animal Care and Use Committee (no. 1705001576). Thirty Holstein heifer calves (4-7 wk of age) were housed outdoors in individual calf hutches $(2.13 \mathrm{~m}$ long $\times 1.17 \mathrm{~m}$ wide $\times 1.35 \mathrm{~m}$ high $)$ with outside runs $(1.83 \mathrm{~m}$ long $\times 1.22 \mathrm{~m}$ wide $)$ and were managed by Purdue University Dairy Research and Education Center staff according to standard farm protocols. Approximately $2 \mathrm{~L}$ of milk was provided to calves twice daily at approximately 0600 and $1600 \mathrm{~h}$. Supplemental starter feed and water were provided in buckets placed inside each calf hutch and refilled as needed twice daily at similar times. Pine shavings were provided as bedding and replaced in the morning every 2 to $3 \mathrm{~d}$ unless inclement weather dictated a bedding change to ensure a dry surface for the calf. All aspects of the current study occurred between July and October 2017.

Each calf was randomly allocated to 1 of 3 treatments: (1) sham disbud (SHAM; $\mathrm{n}=10$ ), (2) disbud with pain mitigation (MED; $\mathrm{n}=10$ ), or (3) disbud without pain mitigation (NoMED; $\mathrm{n}=10$ ). Calves 
that underwent hot-iron disbudding on $\mathrm{d} 0$ of the experimental procedure were given either no pain mitigation (NoMED) or a cornual nerve block under each horn bud $(5 \mathrm{~mL}$ of injectable $20 \mathrm{mg} / \mathrm{mL} 2 \%$ lidocaine $\mathrm{HCl}$ for each horn bud; Vedco Inc., St. Joseph, MO) and $1 \mathrm{mg}$ of meloxicam $/ \mathrm{kg}$ of BW (15-mg oral meloxicam tablets; Patterson Veterinary Inc., Greely, CO) approximately $15 \mathrm{~min}$ before the disbudding procedure (MED). The calves in the SHAM treatment group were treated similarly on d 0 of the experimental procedure (see experimental procedures below); however, they were not disbudded until after they were taken off the study. The experimental procedure was conducted with groups of 3 to 4 calves over 8 repetitions. At least 1 calf per disbudding treatment was included in each repetition.

All calves underwent a period of acclimation to the study personnel, halter rope, and heart rate monitors before the start of the experimental procedure. Once a day for $2 \mathrm{~d}$, study personnel entered the outdoor portion of the hutch area between 0700 and $1000 \mathrm{~h}$ and restrained the calf for approximately 5 min using a halter rope. The study personnel checked the calf's heart rate via hand palpation before applying Spectra electrocardiograph gel (Parker Laboratories Inc., Fairfield, NJ) and a fitting a heart rate (HR) monitor (Polar H10; Polar Electro Oy, Kempele, Finland) for a period of $60 \mathrm{~min}$. Following the acclimation period, the calf was once again restrained using a halter rope and the HR monitor was removed. The hair immediately behind the forelegs of each calf was clipped before the first acclimation period to ensure direct contact between the HR monitor belt and the calf's skin for the remainder of the study.

Following the acclimation period, each calf underwent a 2-h baseline HRV measurement period ( $\mathrm{d}-1$ of the experimental procedure; between 0700 and $1000 \mathrm{~h}$ ). Immediately before data collection, calves were restrained with a halter rope and the calf's HR was quickly checked via hand palpation before placing the HR monitor belt over the palpation area. Electrocardiograph gel was applied to the HR monitor belt to improve signal conduction before securing the HR monitor belt, HR monitor, and telemetric data recorder (Polar V800 Sport Watch; Polar Electro Oy) to the calf with flexible veterinary wrap (VetWrap; 3M, Maplewood, MN). Following the 2-h baseline HRV measurement, the HR monitor belt, HR monitor, and data recorder were removed from the calf.

On d 0 of the experimental procedure, all calves were fit with an HR monitor and underwent treatment between 0700 and $1000 \mathrm{~h}$. Briefly, the disbudding procedure for MED and NoMED calves consisted of 1 study personnel restraining the calf's body while another per- sonnel stood over the calf's neck to limit movement of the calf's head and perform the procedure. A hot-iron dehorner (Express gas dehorner; The Coburn Company Inc., Whitewater, WI) was applied to the horn bud area for approximately 5 to $7 \mathrm{~s}$ to detach and cauterize the surrounding skin and dermis before removing the bud with the dehorner. Sham-disbudded calves received no analgesic treatment and underwent a sham disbudding procedure, where the personnel conducting the study simulated the process of hot-iron disbudding using the large end of a plastic balling gun. All calves were then left alone in their hutches and HRV measurement took place for $8 \mathrm{~h}$ posttreatment. On d 1, 3, and 5 of the experimental procedure, $2 \mathrm{~h}$ of HRV data were collected from each calf between 0700 and $1000 \mathrm{~h}$ to evaluate temporal changes in HRV following disbudding.

Two video cameras (LBV2723B; Lorex Technology Inc., Markham, ON, Canada) were used to record calf behavior both inside and outside each calf's hutch using a digital video system (Geo Vision VMS Software; Geo Vision Inc., Tapei, Taiwan). Posture (standing and lying) was recorded continuously for the entirety of HRV collection to identify periods of inactivity for HRV data selection and analysis.

Heart rate variability data sets were reviewed following data collection, and a single 5-min data set was identified for each calf from each HRV measurement period (on $\mathrm{d}-1,0,1,3$, and 5 ). Heart rate variability data from d 0 (treatment) of the experimental procedure were taken during the last 2 to $4 \mathrm{~h}$ of HRV collection, as this was the period of time when calves were the least disturbed by experimental personnel. This is also a critical point in which the lidocaine cornual nerve block would have diminished. Heart rate variability data from d 1, 3, and 5 were taken at approximately 24, 72, and $120 \mathrm{~h}$ postdisbudding, respectively. Little to no interaction (other than fitting HR equipment) with study personnel took place at these times. All data sets occurred during times of inactivity (lying) and contained less than $10 \%$ erroneous beats. All errors were identified and edited manually using previously published guidelines (Marchant-Forde et al., 2004).

The current study used several nonlinear (SampEn, DFA $\alpha_{1}, \%$ DET, \%REC, Lmean; Table 1) measures that were quantified using available HRV analysis software (Kubios HRV Standard, Kubios Oy; RHRV package in $\mathrm{R}$ 3.3.3, R Foundation for Statistical Computing, Vienna, Austria). Before analysis, data used for measuring SampEn and DFA $\alpha_{1}$ were detrended (first-order differencing) to obtain stationarity. An embedding dimension of 2 inter-beat intervals and a threshold of 0.15 $\times$ standard deviation were used for SampEn analysis. A range of 4 to 16 heartbeat intervals was used for $\mathrm{DFA}_{1}$. 
Percentage determinism (\%DET), \%REC, and Lmean were obtained via recurrence quantification analysis (RQA). Briefly, RQA allows data in multidimensional space to be visualized using a 2-dimensional plot (Eckmann et al., 1987). Recurring points, patterns, and pattern trajectories within the data can then be visualized and quantified using several measures such as \%DET, \%REC, and Lmean. An average time delay parameter $(\tau)$ of 7 for all data sets was determined using the average mutual information method (AMI; "mutual" command in the "tserieschaos" package; R Foundation for Statistical Computing). An average embedding dimension $(m)$ of 5 was selected in a similar manner for all data sets using the false nearest neighbors method ["FNN" command in the "fractal" package; R Foundation for Statistical Computing; parameters: dimension $=15$, lag = determined from AMI, false neighbor Euclidean distance tolerance $\left(\mathrm{R}_{\text {tol }}\right)=14$, neighbor tolerance based on attractor size $\left.\left(A_{t o l}\right)=2\right]$. A radius of 20 beats was used, so the majority of data values had a \%REC value between 1 and 5\% (Wallott, 2017).

Data were analyzed using a linear mixed model (PROC GLIMMIX) with repeated measures in SAS version 9.4 (SAS Institute Inc., Cary, NC). Calf nested within treatment was the experimental unit. Each of the nonlinear HRV measures were used as dependent variables in individual models. Treatments (MED, NoMED, SHAM), time (HRV: d 0, 1, 3, 5), and their interaction (treatment $\times$ time) were used as independent fixed factors. Weight, age, baseline HRV measurement (d -1), and treatment month (July, August, September, October) were also included as covariates in the model. Data sets not meeting the model assumptions (residual normality and homogeneity of variance) were transformed (log transform: \%REC; square root transform: SampEn) as needed before analysis. A KenwardRoger degrees of freedom approximation was used for all analyses. Multiple comparisons were evaluated using Tukey's honestly significant difference test. A statistically significant result was characterized as having an $\alpha$-level $\leq 0.05$. All results are presented as least squares means \pm standard error; however, results from data sets that were transformed before analysis are presented in the results and figures as back-transformed least squares means \pm approximated standard error obtained via the delta method. Data sets from 5 calves were not included in the analysis due to extensive HRV data error. Therefore, all results are based on data from 9 SHAM calves, 8 MED calves, and 8 NoMED calves.

Sample entropy was lower on d $0(1.08 \pm 0.05 \mathrm{~ms})$ compared with d $1\left(1.45 \pm 0.06 \mathrm{~ms} ; t_{29}=-3.05 ; P=\right.$ $0.02)$ and $\mathrm{d} 3\left(1.59 \pm 0.06 \mathrm{~ms} ; t_{33.64}=3.78 ; P=0.004\right)$ due to a numerical decrease in SampEn exhibited by NoMED and MED calves on d 0, whereas SampEn in
SHAM calves remained relatively consistent throughout the study period (Figure 1a). However, no differences between treatments were detected $\left(F_{2,21.63}=2.93 ; P=\right.$ 0.08; Figure 1a).

Calves disbudded with pain mitigation exhibited greater $\mathrm{DFA}_{1}$ than SHAM calves $(1.31 \pm 0.07 \mathrm{AU}$ vs. $1.03 \pm 0.08 \mathrm{AU} ; t_{20.62}=2.60 ; P=0.04$; Figure $\left.1 \mathrm{~b}\right)$. However, NoMED calves displayed an intermediate response and did not differ from MED calves $\left(t_{19.18}=\right.$ $0.39 ; P=0.92)$ or SHAM calves $\left(t_{20.69}=1.98 ; P=0.14\right)$. Detrended fluctuation analysis values were greater on $\mathrm{d}$ $0(1.36 \pm 0.05 \mathrm{AU})$ compared with d $1(1.15 \pm 0.07 \mathrm{AU}$; $\left.t_{26.11}=3.06 ; P=0.02\right)$ and $\mathrm{d} 3\left(1.10 \pm 0.06 \mathrm{AU} ; t_{33.59}\right.$ $=3.38 ; P=0.01$ ), which was largely due to increased DFA $\alpha_{1}$ exhibited by NoMED and MED calves on $\mathrm{d} 0$ (Figure 1b).

Percentage determinism was greater on d 0 compared with $\mathrm{d} 1\left(60.0 \pm 4.1\right.$ vs. $46.4 \pm 4.3 \% ; t_{40.38}=2.72 ; P=$ 0.04 ) due to a numerical increase in \%DET exhibited by NoMED and MED calves on d 0 (Figure 1c). Additionally, \%DET for all calves was lower on d 1 (46.4 $\left.\pm 4.3 \% ; t_{60.11}=-3.24 ; P=0.01\right)$ and $\mathrm{d} 3(52.5 \pm 4.3 \%$; $\left.t_{15.32}=-2.86 ; P=0.05\right)$ compared with d $5(67.1 \pm$ $5.1 \%)$. Percentage determinism did not differ by treatment $\left(F_{2,21.86}=2.29 ; P=0.13\right)$, and no interaction between treatment and time was detected $\left(F_{6,45.51}=\right.$ 1.89; $P=0.10)$.

No effect of treatment $\left(F_{2,16.47}=0.92 ; P=0.42\right)$, time $\left(F_{3,39.4}=2.83 ; P>0.05\right.$ after adjustment for multiple comparisons), or the treatment $\times$ time interaction $\left(F_{6,39.4}=1.01 ; P=0.4313\right)$ was detected for $\%$ REC (Figure 1d). Similarly, no effect of treatment $\left(F_{2,17.15}=\right.$ $1.63 ; P=0.23)$, time $\left(F_{3,24.92}=2.89 ; P=0.06\right)$, or the treatment $\times$ time interaction $\left(F_{6,28.34}=2.77 ; P>0.05\right.$ after adjusting for multiple comparison) was detected for Lmean (Figure 1e).

In the current study, nonlinear HRV measures were used to evaluate correlational, structural, and organizational aspects of the data in response to disbudding. Surprisingly, the only treatment difference observed in the current study occurred between the SHAM and MED treatment groups in relation to $D F A \alpha_{1}$, which is based in fractal theory and can be used for evaluating stress in physiological systems. Fractals, which are present in multiple natural phenomena including HR, are objects or quantities that display statistical self-similarity at different levels of magnification (Mandelbrot, 1982). However, in the case of physiological systems during times of stress or illness, these statistical similarities at different magnifications begin to diminish, and long-term correlations in the data start to break down (Goldberger et al., 2002a). Detrended fluctuation analysis evaluates changes in long-term correlation by measuring the relationship between HRV fluctuations 


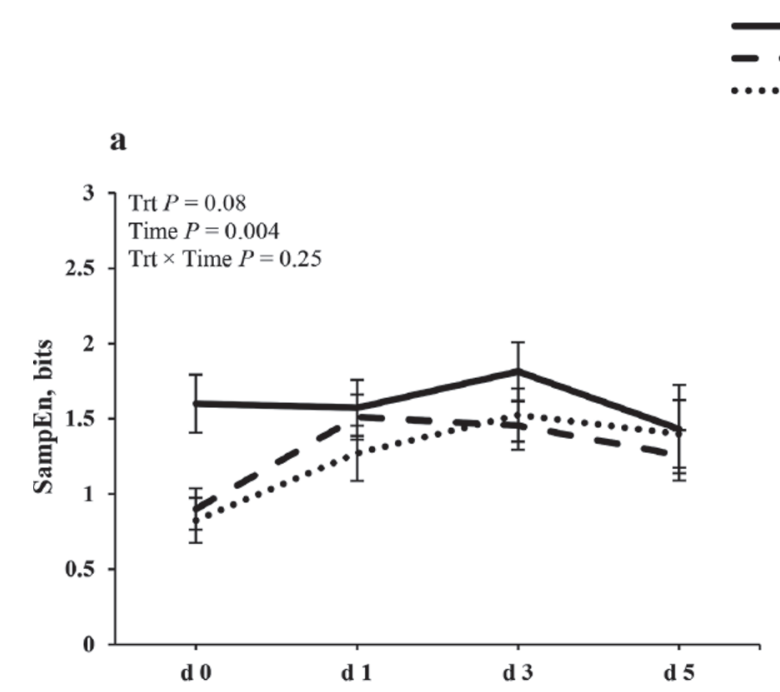

\section{SHAM \\ MED \\ ... NoMED}

\section{d}

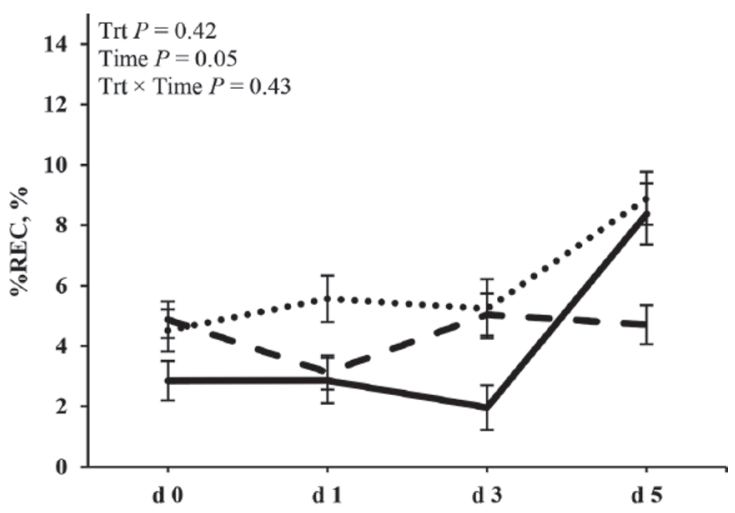

b

e
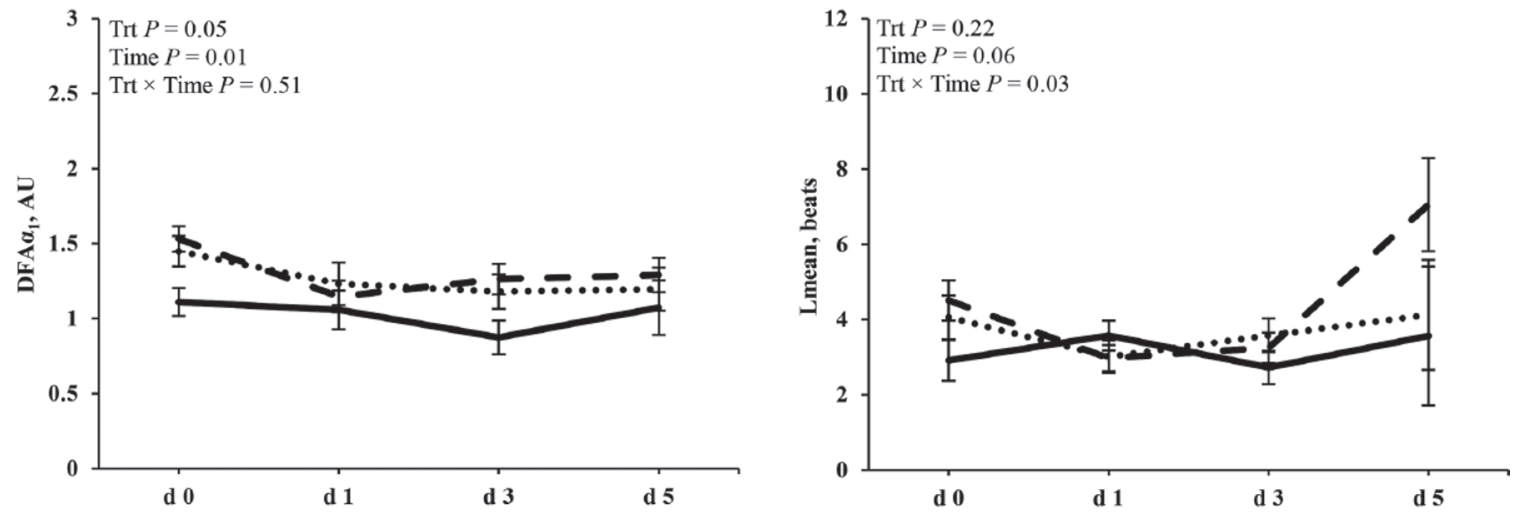

c

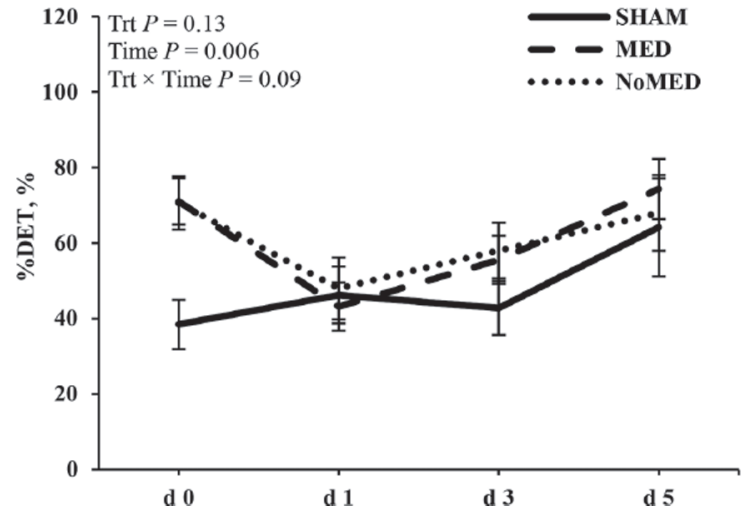

Figure 1. (a) Back-transformed least squares means \pm approximated SE of sample entropy (SampEn) over the 5-d experimental period by treatment. (b) Least squares means $\pm \mathrm{SE}$ of detrended fluctuation analysis $\left(\mathrm{DFA} \alpha_{1}\right)$ over the 5 -d experimental period by treatment. (c) Least squares means $\pm \mathrm{SE}$ of percentage determinism (\%DET) over the 5-d experimental period by treatment. (d) Back-transformed least squares means \pm approximated SE of percentage recurrence (\%REC) over the 5-d experimental period by treatment. After adjustment for multiple comparisons, no differences were observed for time $(P>0.05)$. (e) Least squares means $\pm \mathrm{SE}$ of mean length of diagonal lines in a recurrence plot (Lmean) over the 5-d experimental period by treatment. After adjustment for multiple comparisons, no differences were observed for time or the treatment $\times$ time interaction $(P>0.05)$. Disbudding took place on d 0. Calves in the MED treatment (Trt) group received lidocaine and meloxicam before the disbudding procedure. Calves in the NoMED treatment group did not receive any pain mitigation before the disbudding procedure. Disbudding was only simulated on SHAM calves. 
and scale of measurement over several different data lengths. The integrated HRV data set is first divided into a series of boxes containing $n$ heartbeats. Within each box, a least squares line is fit, the data are detrended, and the root mean squared deviation is calculated. Once this procedure has been carried out for all boxes of a particular length, an average value is plotted onto a log-log graph. This procedure is then repeated for the remaining boxes of different lengths, and a slope of best fit is applied to the log-log graph, which returns a scaling exponent $\left(\alpha_{1}\right)$. In general, scaling exponents close to 1 indicate long-term correlations within the data, which is often viewed as an indicator of health in complex human physiological systems (Goldberger et al., 2002b). Scaling exponents closer to 1.5 indicate shorter-term correlations in the data and therefore may be an indicator of increased stress in physiological systems (Goldberger et al., 2002a).

Very few studies have investigated nonlinear HRV measures in dairy cattle; however, a single study evaluating nonlinear HRV in response to lameness found that cows categorized as lame had $\mathrm{DFA} \alpha_{1}$ scaling exponents closer to 1 , which conflicts with the results reported here and in the human literature (Goldberger et al., 2002a; Kovács et al., 2015). In the current study, MED calves had a scaling exponent closer to 1.5, indicating short-term correlations indicative of greater stress, whereas SHAM calves exhibited long-term correlational structure with a scaling factor close to 1 . The DFA $\alpha_{1}$ responses exhibited by NoMED calves were numerically similar to those of MED calves throughout the experimental period in contrast with SHAM calves, providing some evidence that the meloxicam may not have been sufficient for alleviating pain-related stress throughout the experimental period.

One concern related to the use of nonlinear HRV measures is that their underlying physiological interpretations are not well known in any species, whereas the autonomic interpretations of linear HRV measures are more established (Sassi et al., 2015). However, the usefulness of nonlinear HRV measures for evaluating stressed states is apparent when used in comparison with a control group under experimental conditions (Sassi et al., 2015). Additionally, measures such as $\mathrm{DFA} \alpha_{1}$ have been shown to be powerful predictors of heart-related stress and mortality in humans (Tapanainen et al., 2002). Nonetheless, although DFA $\alpha_{1}$ seems to be a useful indicator of stress in both humans and animals, directionality of the scaling factor and its interpretation in dairy cows in response to painful procedures and states remains unclear. Therefore, further study of $\mathrm{DFA}_{1}$ and its relationship with pain-related stress in dairy calves is needed.
Although the $\mathrm{DFA}_{1}$ results in the current study seem to suggest that the MED calves experienced more pain-related stress compared with the SHAM calves, it's possible that meloxicam artificially altered the HRV of MED calves in a manner similar to the NoMED treatment group. However, previous work evaluating linear HRV measures after disbudding found no effect that would suggest that meloxicam led to artificial alterations in autonomic function (Stewart et al., 2009). One additional alternative explanation may be that the observed change in $\mathrm{DFA} \alpha_{1}$ by the MED treatment group is a result of sympathetic activation during wound healing. Sympathetic nerve fibers innervate the dermis and play a critical role in blood circulation, lymphatic function, and regulation of sweat glands, apocrine glands, and hair follicles (Ashrafi et al., 2016). In response to a soft tissue wound, several neuropeptides are released by the sympathetic and sensory nerve fibers in the dermis that lead to inflammation, angiogenesis, and remodeling of the soft tissue (Ashrafi et al., 2016). Although it is not clear whether HRV is also affected by the increase in sympathetic nerve fiber activity during wound healing, this confounding effect would prohibit HRV from being useful for evaluating pain severity if that were the case. Future studies on the topic should evaluate the effect of sympathetic activity during wound healing on HRV.

\section{ACKNOWLEDGMENTS}

The authors thank John Townsend (Purdue University, West Lafayette, IN); Mike Grott, Gary Wernert, and the Purdue Dairy Farm staff (Purdue University); and Victoria Raber, Stacey Enneking, Ryan Kilgore, Nichole Chapel, Kouassi Kpodo, Jaquelyn Jacobs, Alexis Weldon, Amelia Laub, and Kelsey Clark (USDA-Agricultural Research Service Livestock Behavior Research Unit, West Lafayette, IN) for their help on this project. Mention of trade names or commercial products in this article is solely for the purpose of providing specific information and does not imply recommendation or endorsement by the USDA. No conflicts of interest, financial or otherwise, are declared by the authors.

\section{REFERENCES}

Ashrafi, M., M. Baguneid, and A. Bayat. 2016. The role of neuromediators and innervation in cutaneous wound healing. Acta Derm. Venereol. 96:587-594. https://doi.org/10.2340/00015555-2321.

Billman, G. E. 2013. The $\mathrm{LF} / \mathrm{HF}$ ratio does not accurately measure cardiac sympatho-vagal balance. Front. Physiol. 4:26. https://doi .org/10.3389/fphys.2013.00026.

Dua, S., X. Du, S. V. Sree, and T. Ahamed. 2012. Novel classification of coronary artery disease using heart rate variability analy- 
sis. J. Mech. Med. Biol. 12:1240017. https://doi.org/10.1142/ S0219519412400179.

Eckmann, J. P., S. Oliffson Kamphorst, and D. Ruelle. 1987. Recurrence plots of dynamical systems. Europhys. Lett. 4:973-977. https://doi.org/10.1209/0295-5075/4/9/004.

Erdmann, S., E. Mohr, M. Derno, A. Tuchscherer, C. Schäff, S. Börner, U. Kautzsch, B. Kuhla, H. M. Hammon, and M. Röntgen. 2018. Indices of heart rate variability as potential early markers of metabolic stress and compromised regulatory capacity in dried-off high-yielding dairy cows. Animal 12:1451-1461. https://doi.org/10 $.1017 /$ S1751731117002725.

Faulkner, P. M., and D. M. Weary. 2000. Reducing pain after dehorning in dairy calves. J. Dairy Sci. 83:2037-2041. https://doi.org/10 .3168/jds.S0022-0302(00)75084-3.

Goldberger, A. L., L. A. N. Amaral, J. M. Hausdorff, P. Ch. Ivanov, C.-K. Peng, and H. E. Stanley. 2002a. Fractal dynamics in physiology: Alterations with disease and aging. Proc. Natl. Acad. Sci. USA 99(Suppl. 1):2466-2472. https://doi.org/10.1073/pnas .012579499 .

Goldberger, A. L., C.-K. Peng, and L. A. Lipsitz. 2002b. What is physiological complexity and how does it change with aging and disease? Neurobiol. Aging 23:23-26. https://doi.org/10.1016/ S0197-4580(01)00266-4.

Hagen, K., J. Langbein, C. Schmied, D. Lexer, and S. Waiblinger. 2005. Heart rate variability in dairy cows - influences of breed and milking system. Physiol. Behav. 85:195-204. https://doi.org/10 .1016/j.physbeh.2005.03.019.

Heinrich, A., T. F. Duffield, K. D. Lissemore, and S. T. Millman. 2010. The effect of meloxicam on behavior and pain sensitivity of dairy calves following cautery dehorning with a local anesthetic. J. Dairy Sci. 93:2450-2457. https://doi.org/10.3168/jds.2009-2813.

Heinrich, A., T. F. Duffield, K. D. Lissemore, E. J. Squires, and S. T. Millman. 2009. The impact of meloxicam on postsurgical stress associated with cautery dehorning. J. Dairy Sci. 92:540-547. https:/ /doi.org/10.3168/jds.2008-1424.

Kovács, L., F. L. Kézér, V. Jurkovich, M. Kulcsár-Huszenicza, and J. Tözsér. 2015. Heart rate variability as an indicator of chronic stress caused by lameness in dairy cows. PLoS One 10:e0134792. https://doi.org/10.1371/journal.pone.0134792.

Lake, D. E., J. S. Richman, M. P. Griffin, and J. R. Moorman. 2002 Sample entropy analysis of neonatal heart rate variability. Am. J. Physiol. Regul. Integr. Comp. Physiol. 283:R789-R797. https:// doi.org/10.1152/ajpregu.00069.2002.

Mandelbrot, B. B. 1982. The Fractal Geometry of Nature. Freeman, San Francisco, CA.

Marchant-Forde, R. M., D. J. Marlin, and J. N. Marchant-Forde. 2004. Validation of a cardiac monitor for measuring heart rate variability in adult female pigs: accuracy, artefacts and editing. Physiol. Behav. 80:449-458. https://doi.org/10.1016/j.physbeh.2003.09.007.

Mohr, E., J. Langbein, and G. Nürnberg. 2002. Heart rate variability: A noninvasive approach to measure stress in calves and cows. Physiol. Behav. 75:251-259. https://doi.org/10.1016/ S00319384(01)00651-5.

Parois, S. P., F. A. Cabezón, A. P. Schinckel, J. S. Johnson, R. M. Stwalley, and J. N. Marchant-Forde. 2018. Effect of floor cooling on behavior and heart rate of late lactation sows under acute heat stress. Front. Vet. Sci. 5:223. https://doi.org/10.3389/fvets.2018 .00223 .

Petrie, N. J., D. J. Mellor, K. J. Stafford, R. A. Bruce, and R. N. Ward. 1996. Cortisol responses of calves to two methods of disbudding used with or without local anaesthetic. N. Z. Vet. J. 44:9-14. https://doi.org/10.1080/00480169.1996.35924.

Pincus, S. 1995. Approximate entropy (ApEn) as a complexity measure. Chaos 5:110-117. https://doi.org/10.1063/1.166092.

Rea, P. 2016. Overview of the Nervous System. In: Essential Clinically Applied Anatomy of the Peripheral Nervous System in the Head and Neck. Academic Press. London, England. p. 1-20.

Sassi, R., S. Cerutti, F. Lombardi, M. Malik, H. V. Huikuri, C. K. Peng, G. Schmidt, and Y. Yamamoto. 2015. Advances in heart rate variability signal analysis: joint position statement by the eCardiology ESC Working Group and the European Heart Rhythm Association co-endorsed by the Asia Pacific Heart Rhythm Society. Europace 17:1341-1353. https://doi.org/10.1093/europace/ euv015.

Stewart, M., K. J. Stafford, S. K. Dowling, A. L. Schaefer, and J. R. Webster. 2008. Eye temperature and heart rate variability of calves disbudded with or without local anesthetic. Physiol. Behav. 93:789-797. https://doi.org/10.1016/j.physbeh.2007.11.044.

Stewart, M., J. M. Stookey, K. J. Stafford, C. B. Tucker, A. R. Rogers, S. K. Dowling, G. A. Verkerk, A. L. Schaefer, and J. R. Webster. 2009. Effects of local anesthetic and a nonsteroidal antiinflammatory drug on pain responses of dairy calves to hot-iron dehorning. J. Dairy Sci. 92:1512-1519. https://doi.org/10.3168/jds.2008-1578.

Tapanainen, J. M., P. E. B. Thomsen, L. Køber, C. Torp-Pedersen, T. H. Mäkikallio, A. M. Still, K. S. Lindgren, and H. V. Huikuri. 2002. Fractal analysis of heart rate variability and mortality after an acute myocardial infarction. Am. J. Cardiol. 90:347-352. https: //doi.org/10.1016/S0002-9149(02)02488-8.

USDA. 2018. Dairy 2014, Health and Management Practices on U.S Dairy Operations, 2014. USDA-APHIS-VS-CEAH-NAHMS. Fort Collins, CO.

Wallott, S. 2017. Recurrence quantification analysis of processes and products of discourse: A tutorial in R. Discourse Process. 54:382405. https://doi.org/10.1080/0163853X.2017.1297921. 